\title{
Test yourself: answer - deep benign fibrous histiocytoma
}

\author{
Kerry L. Thomas • Evita Henderson-Jackson • \\ Jamie T. Caracciolo
}

Published online: 14 February 2015

(C) ISS 2015

\section{Discussion}

An AP radiograph shows a radiodense, superficial soft tissue mass in the proximal medial thigh without mineralization or femoral involvement (Fig. 1). Contrast-enhanced CT (Fig. 2) and MRI (Fig. 3) demonstrate a well-defined, smoothly marginated superficial soft tissue mass above the investing muscular fascia with an intervening fat plane and avid contrast enhancement with increased vascularity. While mostly homogenous, the mass has internal necrosis and hemorrhage with central vascularity. There is no definite hypointense pseudocapsule or T2-hyperintense peritumoral edema on MRI. Clinically, there was focal skin ulceration and eschar at the site of bleeding, possibly due to skin stretching in the setting of rapid enlargement. Given concern for hemorrhage, excisional rather than percutaneous needle biopsy was performed with electrocautery dissection around the mass to the underlying fascia, which was not involved.

Histopathology demonstrates a well-circumscribed spindle cell neoplasm without fibrous capsule/pseudocapsule exhibiting storiform architecture with focally striking branching hemangiopericytoma-like vessels arising in the subcutis and deep dermis (Fig. 4); focal ulceration of epidermis without epidermal tumor involvement. Spindle cells are

The case presentation can be found at doi: 10.1007/s00256-015-2112-x

K. L. Thomas $\cdot$ J. T. Caracciolo $(\bowtie)$

Department of Diagnostic Imaging, Moffitt Cancer Center,

Tampa, FL, USA

e-mail: jamie.caracciolo@moffitt.org

E. Henderson-Jackson

Department of Anatomic Pathology, Moffitt Cancer Center,

Tampa, FL, USA evenly distributed without pleomorphism, atypia, foamy histiocytes, or giant cells; the necrosis present is likely due to central infarction. Specimen margins were negative for tumor. Ischemic-like necrosis was noted at $20 \times$ and bland spindle cells without nuclear pleomorphism or hyperchromasia and rare mitoses at $40 \times$.

Benign fibrous histiocytoma (BFH) is a superficial (relative to the investing fascia of the compartmental musculature) soft tissue neoplasm comprised of bland fibroblasts arranged in a storiform pattern with variable giant cells and foamy histiocytes $[1,2]$. Cutaneous BFH (aka dermatofibroma or dermal histiocytoma) arises in the dermis with several histologic variants described [1,2], while deep BFH arises in the subcutis and may histologically resemble cellular-type cutaneous BFH, but the presence of diffuse storiform architecture allows distinction. Forty percent demonstrate hemangiopericytoma-like changes with staghorn-like thin-walled branching vessels. Deep BFH often stains positive for CD34, unlike cutaneous BFH $[3,4]$.

Cutaneous BFH typically presents as a small $(<2 \mathrm{~cm})$, tanbrown, firm, painless skin nodule in the extremities of young adults [2]. Deep BFH also commonly occurs in the extremities, but typically achieves larger size $(0.5-25 \mathrm{~cm}$; median $3 \mathrm{~cm}$ ). Although benign, deep BFH carries increased risk for local recurrence, up to $22 \%$ in one series, and rare metastases have been reported [4]. Sampling with percutaneous or excisional biopsy is needed when lesions are large or necrotic to distinguish BFH from superficial malignant neoplasms such as undifferentiated pleomorphic sarcoma (UPS), cutaneous leiomyosarcoma (LMS), and dermatofibrosarcoma protuberans (DFSP). Pathologically, UPS may be differentiated from BFH by the presence of marked pleomorphism and numerous atypical mitoses. Cutaneous LMS are predominantly well-differentiated smooth muscle tumors exhibiting intersecting fascicular architecture. Like BFH, DFSP displays 
a uniform cellular population with storiform architecture, but DFSP lacks overlying epidermal hyperplasia and typically demonstrates highly infiltrative margins [5].

A large retrospective analysis performed at the Armed Forces Institute of Pathology showed that soft tissue tumors of the lower extremity in young adults (26-35 years) are more often benign than malignant. Over a 10 -year period, $60 \%$ (534/891) of lower extremity soft tissue tumors were benign with BFH being the most common benign neoplasm [6]. Other benign entities in this demographic and location include non-neoplastic etiologies like hematoma and abscess and neoplasms including nodular fasciitis, lipoma, neurofibroma, and hemangioma [7].

Patient age, clinical history, and physical examination significantly contribute to the preoperative differential diagnosis, while imaging findings including size, margins, and location help narrow the differential. Sarcomas are typically large heterogeneous deep-seated tumors arising below the investing fascia with pseudocapsule and peritumoral edema [7]. Conversely, benign tumors tend to be small homogeneous superficial lesions without pseudocapsule, reactive zone, or involvement of the investing fascia [7]. However, there is notable overlap in the presentation of benign and malignant diseases. For example, superficial sarcomas certainly occur, often involving the investing fascia; myxomas may demonstrate peritumoral edema; large deep BFH may have a pseudocapsule and internal necrosis; and cutaneous LMS and DFSP may be small, superficial, and well defined [5, 7-9]. With that said, caution in preoperative diagnosis remains paramount [10]. In this case, despite the large size and internal heterogeneity, several imaging features suggested benignity including age, location, smooth margins, absence of fascial involvement, and lack of peritumoral edema $[5,7-10]$. When considering these findings in light of common benign neoplasms of the lower extremity, a confident preoperative diagnosis may be presented which, in turn, may influence surgical approach.

Conflict of interest The authors declare that they have no conflicts of interest.

\section{References}

1. Pierson J, Tam C. Dermatofibroma [Internet]. 2014 [updated 2014 July 15; cited 2014 Oct 22]. Available from: http://www.emedicine. medscape.com/article/1056742.

2. Shankar V. Soft tissue fibrohistiocytic tumors: benign fibrous histiocytoma (superficial) [Internet]. 2014 [updated 27 June 2014; cited 2014 Oct 22]. Available from http://www.pathologyoutlines. com/topic/softtissuebfh.html.

3. Cheng H. Dermatofibroma (fibrous histocytoma) pathology. [Internet]. 2013 [last updated 25 July 2014; cited 5 Jan 2015]. Available at http://www.dermnetnz.org/pathology/dermatofibromapath.html.

4. Gleason BC, Fletcher CDM. Deep "Benign" fibrous histiocytoma: clinicopathologic analysis of 69 cases of a rare tumor indicating occasional metastatic potential. Am J Surg Path. 2008;32(3):354-62.

5. Goldblum JR, Folpe AL, Weiss SW. Enzinger and Weiss's soft tissue tumors. 6th ed. Philadelphia: Elsevier Saunders; 2014.

6. Kransdorf MJ, Murphey MD. Imaging of soft tissue tumors. 2nd ed. Philadelphia: Lippincott, Williams \& Wilkins; 2006.

7. Wu JS, Hochman MG. Soft-tissue tumors and tumorlike lesions: a systematic imaging approach. Radiology. 2009;253(2):297-316.

8. Galant J et al. Grading of Subcutaneous soft tissue tumors by means of their relationship with the superficial fascia on MR imaging. Skeletal Radiol. 1998;27:657-63.

9. Kransdorf M, Meis-Kindblom J. Dermatofibrosarcoma protuberans: radiologic appearance. AJR. 1994;163:391-4.

10. Kransdorf MJ, Murphey MD. Radiologic evaluation of soft tissue masses: a current perspective. AJR. 2000;175:575-87. 